\title{
Viral hepatitis prevention by immunization
}

\author{
Prevenção das hepatites virais através de imunização \\ Cristina Targa Ferreira ${ }^{1}$, Themis Reverbel da Silveira ${ }^{2}$
}

\section{Resumo}

Objetivo: Apresentar uma revisão atualizada e crítica da prevenção das hepatites virais A e B, através de imunização.

Fontes dos dados: Revisão de artigos médicos obtidos através do banco de dados MEDLINE, sendo selecionados os mais atuais e representativos do tema (2000-2006). Foram também pesquisados os sites do Centers for Disease Control and Prevention (CDC) e American Academy of Pediatrics (AAP), da Sociedade Brasileira de Pediatria (SBP) e do Ministério da Saúde do Brasil.

Síntese dos dados: A prevenção das hepatites virais é um enorme desafio para o sistema de saúde pública dos países e das comunidades médica e científica. Os vírus das hepatites ocasionam importante morbimortalidade no mundo, causando doença hepática aguda e crônica. Vacinas altamente eficazes estão disponíveis no mercado para prevenir novas infecções pelos vírus A e B. Entretanto, as hepatites virais $A$ e $B$ continuam a estar entre as doenças preveníveis por vacinas mais comumente notificadas. Neste artigo, revisamos as vacinas usadas para prevenir essas infecções com o objetivo de expandir o conhecimento e o uso da prevenção dessas doenças infecciosas.

Conclusão: Embora as vacinas contra as hepatites A e B sejam recomendadas para vários grupos de risco, a cobertura vacinal estimada ainda é modesta e existem muitas oportunidades perdidas de vacinação. Para que haja diminuição na incidência das hepatites A e B, duas doenças preveníveis por vacinas, é necessário que os médicos incentivem seus pacientes a receber as vacinas.

J Pediatr (Rio J). 2006;82(3 Supl):S55-66: Hepatite A, hepatite $B$, vacinas, imunização.

\section{Introdução}

As hepatites virais constituem um importante problema de saúde pública no Brasil e no mundo. Descobertas e progressos notáveis, em relação à patogênese, prevenção e tratamento, foram feitos nas 3 últimas décadas. 0 desenvolvimento de vacinas para prevenir essas infecções, através da indução de imunidade ativa contra os vírus das hepatites A e B, foi uma das maiores conquistas científicas. Entretanto, morbidade e letalidade decorrentes dessas doenças ainda persistem. Nos EUA, no ano de 2003, 61.000 indivíduos foram infectados pelo vírus da hepatite viral $A$

1. Doutora em Gastroenterologia, Hospital de Clínicas de Porto Alegre, Universidade Federal do Rio Grande do Sul (UFRGS), Porto Alegre, RS.

2. Doutora em Genética. Professora, Faculdade de Medicina, Hospital de Clínicas de Porto Alegre, UFRGS, Porto Alegre, RS.

Como citar este artigo: Ferreira CT, da Silveira TR. Viral hepatitis prevention by immunization. J Pediatr (Rio J). 2006;82(3 Suppl):S55-66.

\section{Abstract}

Objective: To present an updated review and criticism of viral hepatitis $A$ and $B$ prevention by immunization.

Sources of data: Review of medical articles obtained from the MEDLINE database. The most recent and representative articles on the subject (2000-2006) were selected. The Centers for Disease Control and Prevention (CDC), American Academy of Pediatrics (AAP), Brazilian Society of Pediatrics and Brazilian Ministry of Health websites were also researched.

Summary of the findings: Viral hepatitis prevention is an enormous challenge to the public health systems of countries and the medical and scientific communities. Hepatitis viruses produce important morbimortality in the world, causing acute and chronic hepatic disease. There are highly efficient vaccines available on the market to prevent new infections by the $A$ and $B$ viruses. However, $A$ and $B$ viruses continue to be among the most commonly notified diseases preventable by vaccines. In this article, we discuss the vaccines used to prevent these infections, with the aim of expanding knowledge and the practice of prevention of these infectious diseases.

Conclusions: Although the vaccines against $A$ and $B$ hepatitis are recommended for various risk groups, estimated vaccine coverage is still modest and many vaccination opportunities are lost. In order to reduce the incidence of $A$ and $B$ hepatitis, which are preventable by vaccines, it is necessary for physicians to encourage their patients to be vaccinated.

J Pediatr (Rio J). 2006;82(3 Suppl):S55-66: Hepatitis A, hepatitis $B$, vaccines, immunization.

(VHA) e 73.000, pelo da hepatite $B^{1}$. No Brasil, o Ministério da Saúde estima que $15 \%$ da população já esteve em contato com o vírus da hepatite B (VHB) e que, em média, cerca de $60 \%$ dos indivíduos apresentam anticorpo antiVHA ${ }^{2}$. A Organização Pan-Americana da Saúde (OPAS), por outro lado, estima que mais de $90 \%$ da população maior de 20 anos tenha sido exposta ao vírus e avalia a infecção pelo VHA, no Brasil, em aproximadamente 130 casos novos por 100 mil habitantes/ano.

Em 1992, a Organização Mundial da Saúde (OMS) recomendou que todos os países introduzissem vacina da hepatite $B$ nos programas pediátricos de rotina até $1997^{3}$. Mais do que prevenir a hepatite aguda, a finalidade era reduzir a infecção crônica, freqüentemente não aparente em crianças, mas que determina seqüelas graves na vida adulta. Cerca de 80 a $90 \%$ da população mundial reside em países onde a prevalência de portadores é moderada (2-7\%) ou alta $(>7 \%)^{4}$. 
A primeira vacina disponível para prevenir hepatite viral foi contra o VHB, desenvolvida no início dos anos 1980 e feita com plasma humano. Posteriormente, foi substituída por vacinas produzidas através da técnica do DNA recombinante. A proteção contra a hepatite viral A (HVA), através de vacinas, começou a ser utilizada em grande escala apenas no final dos anos 1990. Mais recentemente, vêm sendo disponibilizadas no mercado vacinas contra hepatite viral associando os antígenos dos vírus VHA e VHB, e vacinas combinadas com os antígenos de outros agentes infecciosos. A instituição dos programas globais de imunoprofilaxia foi eficaz, e houve redução significativa das infecções por VHA e VHB em várias regiões ${ }^{2}$. Atualmente, a imunoprofilaxia das hepatites $A$ e $B$ é realizada através de vacinas seguras, disponíveis em praticamente todo o mundo, e as recomendações dos órgãos oficiais de saúde de diferentes países estão bem documentadas.

No Brasil, embora o número de notificações não reflita a incidência verdadeira da infecção, estudos de soroprevalência para hepatite $A$ mostram que a média nacional está situada em torno de $65 \%{ }^{5}$. Dentre as hepatites virais notificadas aos serviços de saúde, aproximadamente metade dos casos é devida ao VHA.

Nos EUA, onde os contatos domiciliares e sexuais com pessoas infectadas eram o principal modo de transmissão da HVA, entre 1996 e 2002, houve redução na incidência da doença, principalmente na faixa dos 5 aos 14 anos. Foi observado, porém, que a incidência da HVA entre as pessoas que viajam para áreas endêmicas e os homens que têm relação sexual com homens aumentou dramaticamente desde $1995^{4,6}$.

Estima-se em 450 milhões o número de portadores crônicos do vírus $B$ no mundo e em cerca de 2 milhões no Brasil. Nos EUA, embora tenha havido grande redução na incidência de hepatite $B$ aguda, principalmente na faixa dos 15 aos 39 anos, esta continua sendo a faixa etária mais afetada pela infecção aguda ${ }^{6}$. Os indivíduos que têm múltiplos parceiros sexuais e são usuários de drogas continuam sendo os principais transmissores da doença naquele país ${ }^{6}$. Estima-se que o risco de desenvolver hepatocarcinoma é 100 vezes maior entre os portadores do VHB, em comparação aos não portadores do vírus, e que 15-20\% daqueles que possuem o VHB poderão morrer prematuramente ${ }^{3}$.

O motivo para revisar e expandir os conhecimentos atuais e as recomendações dessas vacinas baseia-se nas taxas elevadas, em algumas regiões do país, das hepatites virais $A$ e $B$ e a persistência de uma insuficiente proteção dos indivíduos que se tornam infectados e propagam a infecção viral ${ }^{1,4,6,7}$. O objetivo deste artigo é revisar a prevenção das hepatites A e B através da vacinação, relatar as propriedades das vacinas, revisar estudos clínicos e seus resultados e, finalmente, discutir o impacto da introdução dessas vacinas.

\section{A vacina da hepatite $A$}

Em 1978, Provost \& Hillemann ${ }^{8}$, em estudos animais, demonstraram a habilidade de uma vacina, inativada por formalina, em produzir anticorpos protetores contra HVA. A possibilidade de cultivar o vírus em culturas de células tornou possível a fabricação de grandes quantidades da vacina contra VHA, e o uso de imunoensaios sensíveis e de testes de anticorpos neutralizantes viabilizaram o reconhecimento da capacidade imunogênica das diferentes vacinas.

Afortunadamente, o vírus da HVA existe, no mundo todo, como um único sorotipo, com um pequeno grau de variações antigênicas ${ }^{9}$. As evidências atuais mostram que a imunidade, adquirida naturalmente ou através de vacinas inativadas, protege contra as diferentes cepas do vírus $A^{9}$. Os anticorpos resultantes da vacina, apesar de em menor número, têm a mesma capacidade neutralizante dos anticorpos naturalmente produzidos após a infecção. Embora haja heterogeneidade genética entre as diferentes cepas do vírus $\mathrm{A}$, parece não haver nenhuma variação significativa na conformação do epítopo que determina a neutralização do vírus, pois anticorpos policlonais neutralizam o vírus de todos os genótipos ${ }^{9,10}$. Como a imunidade da HVA é principalmente mediada por anticorpos, a vacina necessita apresentar ao sistema imune do indivíduo a conformação epitópica em dose suficiente para induzir a formação de anticorpos neutralizantes. Para conferir proteção durável, os níveis desses anticorpos devem ser consideravelmente maiores do que aqueles adquiridos com imunoglobulinas.

As vacinas produzidas com vírus inativados por formalina, seguras e altamente eficazes, estão há anos disponíveis no mercado: Havrix ${ }^{\circledR}$ (GlaxoSmithKline), Vaqta ${ }^{\circledR}$ (Merck), Avaxim $^{\circledR}$ (Sanofi Pasteur) e Epaxal ${ }^{\circledR}$ (Berna). O seu licenciamento adicionou um importante benefício no armamentário da imunoprofilaxia e, certamente, vem mudando o cenário da prevenção da hepatite $A^{1}$. Na rede pública, a vacina está disponível apenas nos Centros de Referência de Imunobiológicos Especiais (CRIE) e distribuída para grupos específicos de indivíduos. As diferentes vacinas comercializadas no Brasil, com suas apresentações e doses, estão na Tabela $1^{2}$.

Embora as doses e as formulações das vacinas sejam diferentes, tanto o número quanto o intervalo das doses são os mesmos: devem ser administradas em duas doses, com intervalo de 6 meses. Dados ainda limitados indicam que, se esse intervalo entre a primeira e a segunda dose não puder ser observado, não haverá maior comprometimento nas concentrações dos anticorpos. De uma maneira geral, considerando os excelentes resultados obtidos com a vacina, não há necessidade de testes pós-vacinais.

A vacina da HVA é derivada de vírus adaptados em culturas de células, posteriormente purificados e inativados com formalina e finalmente adsorvidos a um adjuvante, geralmente o hidróxido de alumínio.

Estudos clínicos, baseados em milhões de doses administradas em pessoas saudáveis, demonstraram a eficácia protetora e a tolerabilidade das vacinas inativadas ${ }^{11,12}$. Mesmo esquemas de imunização com uma só dose da vacina fornecem proteção imediata ${ }^{13}$. Os níveis de anticorpos anti-VHA, após uma única dose da vacina, são maiores do que os produzidos por imunoglobulina, mas são inferiores àqueles gerados pela infecção natural6,14,15. Após 1 
Tabela 1 - Preparações e doses recomendadas de vacinas contra hepatite A

\begin{tabular}{|c|c|c|c|c|c|}
\hline Tipo da vacina & $\begin{array}{l}\text { Idade } \\
\text { (anos) }\end{array}$ & Dose & $\begin{array}{c}\text { Volume } \\
(\mathrm{mL})\end{array}$ & $\begin{array}{l}\text { Número } \\
\text { de doses }\end{array}$ & $\begin{array}{c}\text { Esquema } \\
\text { (meses) }\end{array}$ \\
\hline Havrix ${ }^{\circledR} *$ & $\begin{array}{l}1-18 \\
>18\end{array}$ & $\begin{array}{c}720 \text { ELISA } \\
1440 \text { ELISA }\end{array}$ & $\begin{array}{l}0,5 \\
1,0\end{array}$ & $\begin{array}{l}2 \\
2\end{array}$ & $\begin{array}{l}0 \text { e } 6-12 \\
0 \text { e } 6-12\end{array}$ \\
\hline Vaqta $^{\circledR}+$ & $\begin{array}{l}1-17 \\
>17\end{array}$ & $\begin{array}{l}25 \mathrm{U} \\
50 \mathrm{U}\end{array}$ & $\begin{array}{l}0,5 \\
1,0\end{array}$ & $\begin{array}{l}2 \\
2\end{array}$ & $\begin{array}{c}0 \text { e } 6-12 \\
0 \text { e } 6\end{array}$ \\
\hline Avaxim $^{\circledR} \neq$ & $>1$ & $160 \mathrm{U}$ & 0,5 & 2 & 0 e $6-18$ \\
\hline 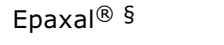 & $>1$ & $500 \mathrm{U}$ & 0,5 & 2 & 0 e 6 \\
\hline \multirow[t]{3}{*}{$\begin{array}{l}\text { Twinrix }^{\circledR} * \\
(\text { Hep A+B) }\end{array}$} & $1-15$ & $\begin{array}{c}360 \text { ELISA + } \\
10 \mu \mathrm{g} \mathrm{de} \\
\text { HBsAg (Hep B) }\end{array}$ & 0,5 & 3 & 0,1 e 6 \\
\hline & $1-5$ & $\begin{array}{c}720 \text { ELISA + } \\
20 \mu \text { de HBsAg } \\
(\text { Hep B) }\end{array}$ & 1,0 & 2 & 0 e $6-12$ \\
\hline & $>16$ & $\begin{array}{c}720 \text { ELISA + } \\
20 \mu \text { de HBsAg } \\
(\text { Hep B) }\end{array}$ & 1,0 & 3 & 0,1 e 6 \\
\hline
\end{tabular}

* GlaxoSmithKline; ${ }^{\dagger}$ MerckSharp \& Dohme; ${ }^{\ddagger}$ Sanofi Pasteur; $§$ Berna Biotech.

mês da primeira dose, > 97\% das crianças e > 95\% dos adultos desenvolvem níveis protetores de anticorpos (definidos como 10 a 20 UI/L) ${ }^{6,16}$. No sétimo mês, ou seja, 1 mês após a segunda dose, virtualmente $100 \%$ das pessoas apresentam níveis protetores de anticorpos anti-VHA. Os estudos clássicos de Innis ${ }^{11}$ e Werzberger ${ }^{12}$, envolvendo 38.175 crianças de 1 a 16 anos e 1.037 crianças de 2 a 16 anos, na Tailândia e em Nova Iorque, mostraram que houve proteção pelas vacinas em 94 e 100\%, respectivamente. Os autores demonstraram que os efeitos colaterais dessas vacinas foram similares, ou menos intensos, que os causados por placebo ou aqueles ocasionados pela vacina da hepatite B. Além disso, os efeitos adversos, exceto febre, diminuem de freqüência com doses sucessivas ${ }^{13,17}$.

A resposta imune à infecção por HVA é complexa, mas, claramente, envolve imunidade celular e humoral, sendo a imunidade celular necessária para que se desenvolva memória imunológica. Vários estudos mostram que a vacina anti-VHA induz imunidade humoral, com níveis de até $100 \%$ de soroconversão. De acordo com modelos de cinética viral, a proteção determinada pela vacina inativada permanece pelo menos por 5 a 10 anos, havendo a possibilidade de continuar protegendo os indivíduos até 20 anos após a vacinação. Van Herck et al. ${ }^{18}$ desenvolveram modelos matemáticos para avaliar a evolução dos anticorpos antiVHA, após administração de duas vacinas inativadas (Havrix e Avaxim). As duas vacinas mostraram resultados semelhantes. Quando os níveis de anticorpos foram maiores de $20 \mathrm{mUI} / \mathrm{mL}$, permanecem pelo menos 10 anos após completar o esquema de vacinação. É oportuno lembrar que os indivíduos respondedores com títulos decrescentes e mesmo indetectáveis podem seguir protegidos, através da resposta anamnéstica antiviral ${ }^{11,13}$. Nesses indivíduos vacinados, confirmando a memória imunológica, foram detectados anticorpos na reexposição ao antígeno ${ }^{19}$. Embora a duração da proteção induzida pela vacina não esteja bem determinada, os modelos matemáticos sugerem que níveis protetores de anticorpos possam persistir por 24 a 47 anos após a segunda dose, administrada 6 a 12 meses após a primeira 20

Cederna et al. ${ }^{19}$ demonstraram proliferação celular em resposta à vacina em 10 pacientes. A vacina induziu uma resposta precoce de proliferação das células $T$, que persistiu por pelo menos 5 meses, e foi acompanhada pela produção de gama-interferon. Essa resposta das células $\mathrm{T}$ vírusespecífica sugere que os indivíduos vacinados apresentam uma reação imune (anamnéstica) precoce, quando da presença do vírus, produzindo rapidamente, e em altos níveis, anticorpos neutralizantes. Assim, cada vez que o indivíduo entra em contato com o vírus, esse fato pode agir como uma dose de reforço da vacina, sugerindo que a memória imunológica está presente nos indivíduos que recebem a vacina inativada contra HVA ${ }^{19}$. Deve-se lembrar que a eficácia da imunoglobulina está relacionada aos anticorpos circulantes, somente, portanto, à imunidade humoral ${ }^{18,19}$.

Nos últimos anos, a incidência da HVA nos EUA tem mostrado uma redução importante com o uso da vacina: de 12 casos/100.000 indivíduos em 1995 para 3,1 casos/ 100.000 em $2002^{4,6,21}$. Essa redução foi maior nos estados onde a vacinação de todas as crianças maiores de 2 anos estava preconizada pelo Advisory Committee for Immunization Practices (ACIP). A partir de 1999, foi observada uma diminuição de $67 \%$ - de 7,2 casos/ 100.000 para 2,4 casos/ 
100.000 em $2002^{4,6}$. Essa redução foi similar em seis outros estados norte-americanos onde a vacina foi recomendada, embora não obrigatória - $69 \%$ de redução, de 6,4 casos/ 100.000 para 2 casos/100.000 em $2002^{4,6}$. Nos outros 33 estados onde a vacina da HVA não foi estabelecida como rotina, as reduções na incidência da doença foram muito menores - 35\% de redução, de 3,4 casos/100.000 em 1999 para 2,2 casos/100.000 em 2002. Foi verificado que a grande redução dos casos de hepatite A, entre 1996 e 2002, ocorreu na faixa dos 5 aos 14 anos ${ }^{4,6}$. Recentemente, houve uma mudança importante nas recomendações do ACIP. Foram aprovadas tanto a orientação para vacinar todas as crianças com idade de 12 a 23 meses quanto a recomendação para incluir a vacina contra a hepatite $A$ no calendário vacinal rotineiro das crianças e adolescentes ${ }^{22}$.

\section{A vacina da HVA em grupos especiais}

Existem várias indicações para a vacinação contra $\mathrm{VHA}^{2,7}$ : hepatopatas crônicos; indivíduos com distúrbios de coagulação; imunodeprimidos; homossexuais de ambos os gêneros; viajantes para zonas endêmicas; familiares de pacientes com hepatite $A$; trabalhadores em creches; profissionais da saúde; indivíduos suscetíveis na vigência de surtos da infecção; residentes em orfanatos, instituições; usuários de drogas ilícitas; trabalhadores em contato com água não tratada; crianças que vivem em áreas de endemicidade intermediária.

Em alguns grupos, como ocorre com outras vacinas, a vacina contra HVA pode não resultar em resposta protetora em todos indivíduos suscetíveis vacinados, principalmente em pacientes imunodeprimidos ${ }^{21}$. Considerando que a vacina da hepatite $A$ é inativada, nenhuma precaução especial necessita ser tomada para vacinar os indivíduos imunodeprimidos 21 .
Alguns estudos mostram que indivíduos adultos infectados pelo HIV apresentam taxas menores de soroproteção, assim como níveis séricos mais baixos de anticorpos antiHVA após a aplicação da vacina ${ }^{23}$. Estudo brasileiro demonstrou que a média geométrica de títulos de anticorpos anti-HVA não diferiu entre as crianças infectadas pelo HIV e aquelas negativas ${ }^{24}$.

A prevalência de anticorpos contra o VHA foi analisada em 352 crianças e adolescentes expostos e/ou infectados pelo HIV, em São Paulo, por Gouvea et al. ${ }^{25}$, tendo sido observada positividade em apenas $26 \%$. Nenhum desses pacientes havia sido vacinado contra a hepatite A. A possibilidade da infecção pelo HIV ser mais grave quando associada ao VHA torna indispensável a profilaxia nesse grupo de pacientes. Alguns autores sugerem que a vacina deva ser oferecida em estágios precoces da infecção pelo HIV26,27. Outros grupos de pacientes imunodeprimidos, como os renais crônicos, dialisados e hemofílicos, já foram estudados e apresentam respostas variáveis, mas com boas taxas de soroconversão $28-31$ (Tabela 2).

O conselho pediátrico da American Liver Foundation 32 recomenda a vacina para crianças com qualquer tipo de doença hepática crônica. Os autores consideram que a HVA pode ser particularmente grave em crianças com imunodeficiências e lembram que o VHA pode servir como gatilho para doenças hepáticas auto-imunes em indivíduos predispostos $28,31,32$. Embora as pessoas com doença hepática crônica não apresentem risco aumentado de contrair HVA, elas parecem ter uma maior propensão de desenvolver complicações e de evoluir para óbito quando contraem a infecção 14,21,23,31. A resposta dos pacientes com doença hepática crônica descompensada é sabidamente menor, com níveis mais baixos de anticorpos e menores taxas de soroconversão, quando comparada àqueles com doença compensada $6,17,33,34$.

Tabela 2 - Taxas de soroconversão e GMT da vacina HVA em grupos especiais

\begin{tabular}{|c|c|c|c|c|c|}
\hline $\begin{array}{l}\text { Estudo } \\
\text { (aplicação intervalo) }\end{array}$ & $\begin{array}{l}\text { Grupo de } \\
\text { pacientes }\end{array}$ & $\begin{array}{l}\text { Idade em } \\
\operatorname{anos}(x)\end{array}$ & $\mathbf{n}$ & $\begin{array}{l}\text { \% soroconversão } \\
\text { (valor de corte) }\end{array}$ & $\begin{array}{l}\text { GMT final } \\
(\mathrm{mUI} / \mathrm{mL})\end{array}$ \\
\hline $\begin{array}{l}\text { Cañero-Velasco et al. }{ }^{29} \\
(\text { IM, } 1-6 \mathrm{~m})\end{array}$ & $\begin{array}{l}\text { Renais crônicos } \\
\text { Síndrome nefrótica }\end{array}$ & $\begin{array}{c}2-9 \\
(5,3)\end{array}$ & 16 & $\begin{array}{c}100 \% \\
(\geq 20 \mathrm{mUI} / \mathrm{mL})\end{array}$ & 361 \\
\hline $\begin{array}{l}\text { Hess et al. }{ }^{23} \\
(\mathrm{IM}, 0,1-6 \mathrm{~m})\end{array}$ & $\begin{array}{l}\text { HIV + } \\
\text { HIV - }\end{array}$ & $\begin{array}{l}21-60 \\
(33,2)\end{array}$ & $\begin{array}{l}26 \\
20\end{array}$ & $\begin{array}{c}76,9 \% \\
100 \% \\
(\geq 20 \mathrm{mUI} / \mathrm{mL})\end{array}$ & $\begin{array}{c}636 \\
1.687\end{array}$ \\
\hline $\begin{array}{l}\text { Santagostinho et al. }{ }^{26} \\
\text { (SC, } 1-6 \text { dias) }\end{array}$ & $\begin{array}{c}\text { Hemofílicos } \\
\text { HIV + } \\
\text { HIV - }\end{array}$ & $\begin{array}{l}1-50 \\
(17)\end{array}$ & $\begin{array}{l}47 \\
66\end{array}$ & $\begin{array}{c}85 \% \\
100 \% \\
(\geq 20 \mathrm{mUI} / \mathrm{mL})\end{array}$ & $\begin{array}{c}503 \\
3.199\end{array}$ \\
\hline $\begin{array}{l}\text { Ferreira et al. }{ }^{35} \\
(\mathrm{IM}, 0 \text { e } 6 \mathrm{~m})\end{array}$ & Síndrome de Down & $\begin{array}{c}1-11 \\
(3,96)\end{array}$ & 49 & $\begin{array}{c}100 \% \\
(>33 \mathrm{mUI} / \mathrm{mL})\end{array}$ & $1.719,86$ \\
\hline
\end{tabular}

$\mathrm{IM}=$ intramuscular; $\mathrm{SC}=$ subcutâneo. 
Recentemente foi avaliada, em Porto Alegre, a resposta à vacina de crianças portadoras de síndrome de Down e de hepatopatias crônicas ${ }^{35-37}$. Foram obtidos resultados satisfatórios de soroconversão, mas com títulos de anticorpos menores do que aqueles das crianças normais da mesma faixa etária. Os resultados encontram-se nas Tabelas 2 e 3.

As taxas de soroconversão 1 mês após as duas doses da vacina contra HVA (esquema 0 e 6 meses) são similares entre adultos saudáveis e indivíduos com hepatite crônica $B$ $(98,2$ versus $97,7 \%)$, com hepatite crônica $C(98,2$ versus $94,3 \%)$ ou com outras hepatopatias $(98,2$ versus $95,2 \%)^{6,17,30}$.

Nebbia et al. ${ }^{38}$ estudaram crianças e adolescentes até 16 anos de idade portadoras de cirrose e observaram uma resposta adequada à vacina da HVA. Os resultados encontram-se na Tabela 3.

\section{Impacto da vacinação anti-HVA}

A situação epidemiológica de uma determinada região não é estática, apresenta mudanças em razão de vários fatores. Dentre eles, talvez o mais importante seja a modificação nas condições socioeconômicas e de higiene 39,40 . Em algumas regiões da América Latina, nos últimos anos, nota-se uma diminuição de casos em faixas etárias menores com deslocamento da curva de soroprevalência da infecção para a idade adulta ${ }^{41}$. Isso ocorre, basicamente, como resultado das melhorias nas condições sanitárias e socioeconômicas das populações.

A existência de vacina contra o VHA viabiliza diminuir substancialmente a incidência da doença, eliminar a transmissão do vírus e, eventualmente, até mesmo erradicar a infecção. A redução na incidência da doença será alcançada através da promoção de altos níveis de imunidade em pessoas que servem como reservatórios do vírus. Essa população, altamente imunizada, diminuirá a incidência da HVA e, presumivelmente, a circulação do vírus.
A possibilidade de implantação da vacina anti-VHA no calendário da rede pública obriga a ponderações especiais. Deve levar em conta, entre outros, os seguintes aspectos: as características epidemiológicas locais, o custo da vacina, as condições de saúde da população e a cobertura vacinal de outras infecções. Por exemplo, um recente estudo soroepidemiológico de escolares indianos, com idade entre 4-18 anos, mostrou que, aos 5 anos de idade, $80 \%$ já apresentavam anticorpo anti-VHA positivo, demonstrando, assim, que a vacinação em massa nessa população (mesmo aos 5 anos) provavelmente não seria custo-efetiva ${ }^{42}$.

Definir as características epidemiológicas da hepatite viral em país de dimensões continentais, como o Brasil, não é fácil. Por outro lado, a elevada percentagem de casos assintomáticos de HVA determina que a soroprevalência seja um indicador epidemiológico melhor do que a incidência da infecção. Assim, Costa-Clemens et al. ${ }^{5}$ estudaram a soroprevalência do VHA, em quatro capitais brasileiras, e mostraram um padrão heterogêneo entre as diferentes regiões. Concluíram que crianças, adolescentes e adultos jovens soronegativos para o anti-HVA, em nosso país, têm um risco similar ao de viajantes para regiões de alta endemicidade, considerando que eles não estão protegidos, além de estarem sob contínuo risco de exposição.

Em Porto Alegre, também foi demonstrado esse padrão heterogêneo, de acordo com as classes sociais, sendo evidenciada a necessidade da vacinação anti-VHA. Crianças suscetíveis (com anticorpo anti-VHA negativo) vivem nas mesmas áreas onde a HVA é comum ${ }^{43}$ (Figura 1). Em estudo semelhante, realizado em São Luís do Maranhão, a prevalência de IgG anti-VHA, de 462 crianças de escolas públicas e privadas, com idades compreendidas entre 7 e 14 anos, foi de 71,5 e $36,5 \%$, respectivamente ${ }^{44}$.

Até o momento, não há estratégias estabelecidas de vacinação anti-VHA em ampla escala nos países da América Latina. Por um lado, nas regiões com elevada endemicidade, é quase impraticável identificar os grupos de risco para

Tabela 3 - Taxas de soroconversão e GMT da vacina HVA em crianças e adolescentes portadores de hepatopatia crônica

\begin{tabular}{|c|c|c|c|c|c|c|}
\hline Estudo & $\begin{array}{l}\text { Grupo de } \\
\text { pacientes }\end{array}$ & $\begin{array}{l}\text { Idade em } \\
\operatorname{anos}(\mathbf{x})\end{array}$ & $\mathbf{n}$ & $\begin{array}{c}\text { Vacina } \\
\text { (meses) }\end{array}$ & $\begin{array}{l}\text { \% soroconversão } \\
(1 \mathrm{~m}-7 \mathrm{~m})\end{array}$ & $\begin{array}{c}\text { GMT final } \\
(\mathrm{mUI} / \mathrm{mL})\end{array}$ \\
\hline \multirow[t]{4}{*}{ Nebbia et al. 38} & Portadores & $2-15$ & 33 & Havrix & $90,9 \%$ & $3.776,8$ \\
\hline & $\mathrm{HbsAg}$ & $(10,7)$ & & 360 UE & $100 \%$ & \\
\hline & 24 hep. crônicos & & & $(0,1$ e 6$)$ & & \\
\hline & 0 cirrose & & & & & \\
\hline \multirow[t]{3}{*}{ Ferreira et al. ${ }^{36}$} & Cirrose & $1-14(4,3)$ & 17 & Havrix & $94-100 \%$ & $1.490,55$ \\
\hline & AVBEH & $10-16(12,4)$ & 7 & 720 UE & $57-100 \%$ & 506,18 \\
\hline & $\begin{array}{c}\text { Auto-imune } \\
\text { Outros }\end{array}$ & $2-15(8,4)$ & 10 & $(0$ e 6$)$ & $60-90 \%$ & 395,44 \\
\hline
\end{tabular}




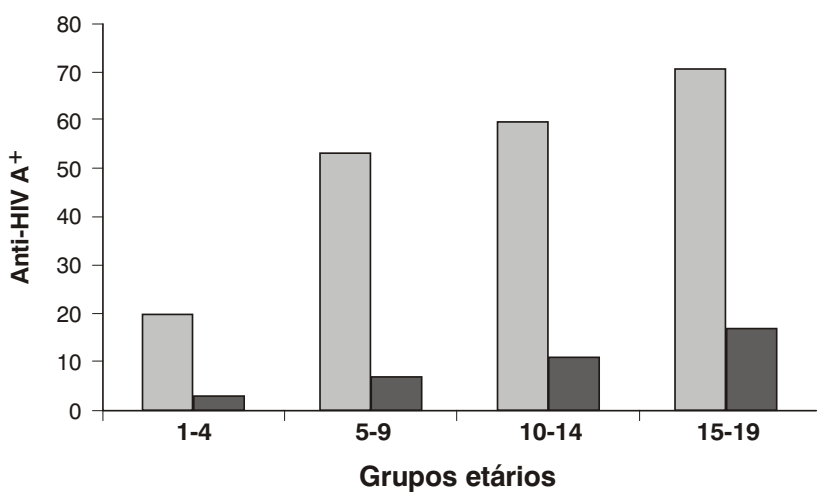

$\square$ Baixo nível socioeconômico $\square$ Alto nível socioeconômico

Figura 1 - Prevalência de anticorpo anti-HVA em dois grupos de nível socioeconômico distintos de Porto Alegre

aquisição da doença. Por outro lado, o elevado custo da vacina torna difícil estender esse benefício a toda população $45-47$.

Israel foi o primeiro país a adotar a vacinação universal para crianças aos 18 e 24 meses, em julho de 1999, e os resultados foram excelentes ${ }^{48}$. Uma análise bem recente do impacto desse programa, 5,5 anos após o seu início, revela que um declínio importante na incidência da doença foi observado em todas as faixas etárias com uma cobertura de $90 \%$ para a primeira dose e de $85 \%$ para a segunda 48 . Os autores sugerem que os programas de imunização universal, desenhados para pré-escolares, são altamente efetivos e factíveis ${ }^{48}$.

Um outro estudo recente, realizado na Austrália, também revelou o impacto da vacinação para crianças indígenas (duas doses feitas aos 18 e 24 meses) ${ }^{49}$. Embora o programa tenha sido desenhado para proteger a população de alto risco, houve um rápido e importante declínio nas taxas de notificação e o benefício logo se estendeu a outros grupos da comunidade ${ }^{49}$.

As crianças em idade pré-escolar devem ser o foco principal das estratégias de imunização, pelo seu papel crítico na transmissão do vírus $21,45-47,50$. Conforme já bem demonstrado por diferentes estudos, a maneira eficaz para diminuir a incidência da doença e até mesmo erradicar a infecção é a vacinação em massa de crianças. Muitas barreiras, no entanto, existem para que essa medida possa ser adotada, entre elas: o crescente número de vacinas na infância, outras prioridades de saúde pública, o custo da vacina e o reduzido número de estudos de custo-efetividade dessa estratégia para o nosso país. Até que isso ocorra, a estratégia aplicada em nosso país continuará sendo a de prevenir a HVA em grupos de risco, respeitando a hierarquia na destinação dos recursos públicos disponíveis. Enquanto a vacinação em massa não ocorrer, vamos continuar assistindo pacientes desenvolver quadros graves de hepatite, incluindo a forma fulminante. Recentemente, demonstramos, em estudo multicêntrico, que aproximadamente $40 \%$ dos casos de insuficiência hepática das crianças na América Latina são devidos à hepatite $A^{51}$.

\section{A vacina da hepatite $B$}

A hepatite causada pelo vírus B é, ainda nos dias atuais, uma das principais causas de doença hepática no mundo. Estudos epidemiológicos revelam uma forte relação entre a infecção pelo VHB, a cirrose e o carcinoma hepatocelular. Calcula-se que em torno de 1 milhão de pessoas morrem por complicações da doença hepática a cada ano1,4,6,7,51,52. A hepatite crônica causada pelo VHB tem se revelado uma doença complexa, de difícil manejo, em função de, entre outros fatores, peculiaridades na relação vírus-hospedeiro, surgimento de mutantes, heterogeneidade viral e diversidade das formas clínicas ${ }^{53}$. Nos EUA, foi estimado que um indivíduo tem $5 \%$ de chance, no decorrer de sua vida, de entrar em contato com o $\mathrm{VHB}^{4,21}$.

As principais finalidades da vacinação contra o VHB são prevenir a doença aguda, impedir a cronificação da hepatopatia e sua evolução para cirrose e/ou hepatocarcinoma e, ainda, contribuir para minimizar a transmissão viral. As características da transmissão do VHB tornam necessário implantar estratégias complexas de vacinação, para que sejam protegidos tanto recém-nascidos quanto adolescentes e adultos. A estratégia utilizada pela OMS, endossada por praticamente todas as outras organizações oficiais para controlar a infecção pelo vírus $\mathrm{B}$, foi a introdução da vacina para todas as crianças ao nascimento 4,54,55.

Nas populações de baixo risco, como na maioria dos estados do Brasil, onde a transmissão primariamente ocorre em indivíduos com mais idade, a imunização dos recém-nascidos é usada para prevenir contaminação no início da vida e a subseqüente cronificação. Sabe-se que o risco de desenvolver a infecção crônica está inversamente relacionado com a idade ${ }^{6,7}$ (Tabela 4). Com a vacinação das crianças no início da vida, diminui também a contaminação horizontal, tão freqüente nos domicílios onde há portador de VHB. Além disso, quando o esquema vacinal é instituído nessa fase da vida, há maior probabilidade da realização de séries completas de vacinação ${ }^{52}$.

Tabela 4 - Idade da infecção em relação à possibilidade de se tornar um portador crônico do vírus da hepatite $\mathrm{B}$

\begin{tabular}{cc}
\hline $\begin{array}{c}\text { Idade da } \\
\text { criança (anos) }\end{array}$ & $\begin{array}{c}\text { Percentual (\%) de crianças } \\
\text { que se tornam portadoras crônicas }\end{array}$ \\
\hline$<1$ & $70-90$ \\
$2-3$ & $40-70$ \\
$4-6$ & $10-40$ \\
$>7$ & $6-10$ \\
\hline
\end{tabular}

O prognóstico da hepatite crônica adquirida na infância permanece incerto. A reativação da replicação viral, ou da doença hepática, pode ocorrer bem mais tarde, na idade adulta, e a soroconversão para anti-HBe nem sempre está associada com o desaparecimento do HBVDNA e/ou com a remissão da hepatopatia. 
Vacinas seguras e eficazes contra HVB estão disponíveis em todo o mundo. Em termos globais, estima-se que mais de 1 bilhão de pessoas já tenham recebido vacina contra hepatite $B$; nos EUA, os cálculos apontam mais de 40 milhões de crianças e de 30 milhões de adultos já vacinados $^{6,55}$. O antígeno utilizado nas vacinas disponíveis atualmente é um HBsAg recombinante, produzido pela inserção de um plasmídeo, contendo o gene do antígeno de superfície, em células de um fungo6,55. O HBsAg, após purificação, é adsorvido a hidróxido de alumínio, sendo que o produto final contém mais de $95 \%$ de proteína $\mathrm{HBsAg}$, menos de $5 \%$ de proteínas derivadas do fungo e nenhum DNA do fungo detectável na vacina. As vacinas Recombivax-HB ${ }^{\circledR}$ são formuladas sem preservativos, e as vacinas Engerix- ${ }^{\circledR}$ são consideradas livres de preservativos, apesar de poderem conter traços de mercúrio após a remoção do timerosal. Essas vacinas sofrem a remoção do timerosal, pós-produção, que é considerado sem efeito biológico ${ }^{6}$. A proteção da vacina contra hepatite $B$ é, pelo menos em parte, derivada da memória imunológica estabelecida. Os níveis de anticorpos derivados da vacina normalmente declinam com o passar do tempo, mas eles permanecem por pelo menos 15 anos após a série completa de vacinação e são reativados, quando necessário, pela memória imunológica. Por esse motivo, doses de reforço não são habitualmente recomendadas, a não ser em alguns grupos especiais, de risco 6,55 .

No âmbito da saúde pública, não se preconiza a avaliação rotineira dos marcadores sorológicos da infecção nos candidatos à vacinação (crianças e adultos), assim como está dispensada a pesquisa de anti-HBs pós-vacinal no soro dos indivíduos imunocompetentes.

As vacinas comercializadas no Brasil, com suas apresentações e doses, são mostradas nas Tabelas 1 e $5^{2}$. Geralmente são administradas em três doses, sendo a segunda e a terceira doses aplicadas 1 e 6 meses após a primeira. Se a série foi interrompida após a primeira dose, a segunda deve ser dada logo que possível, e a terceira pelo menos 2 meses após a segunda. Se apenas a terceira dose estiver faltando, ela deve ser administrada imediatamente. Esquemas alternativos podem ser utilizados da seguinte forma ${ }^{22}$ :

- Vacinação rápida : primeira dose no dia 0 e as outras doses após 1, 2, e reforço aos 12 meses;

- Vacinação acelerada: primeira dose no dia 0 e as outras após 7 e 21 dias e reforço aos 12 meses;

- Esquema de 2 doses: primeira dose no dia 0 e outra 612 meses após (indivíduos especiais).

Testes pós-vacinação são aconselhados para certos indivíduos: as crianças nascidas de mães infectadas (devem ser avaliadas entre os 9 e 15 meses), os profissionais de saúde em contato com sangue e/ou derivados, os pacientes hemodialisados (testar 1 a 2 meses após a última dose da vacina) e os parceiros sexuais de portadores do VHB (testar 1 a 2 meses após a última dose da vacina).

No Brasil, o Programa Nacional de Imunizações (PNI) recomenda que a primeira dose da vacina deve ser administrada na maternidade, nas primeiras 12 horas de vida do recém-nascido. Se os adolescentes (11 a 19 anos) não tiverem comprovação de vacina anterior, deve ser iniciada a série ${ }^{2}$.

A proteção da vacina contra HVB aumenta com o número de doses aplicadas. De maneira geral, essa é uma vacina muito eficaz, com taxas de proteção de $95 \%$, com variações de 80 a $100 \%$ entre aqueles indivíduos que são submetidos ao esquema completo de vacinação. Considera-se proteção quando os títulos de anticorpos ao HBsAg (anti-HBs) são maiores do que $10 \mathrm{mUI} / \mathrm{ml}^{6}$. Na faixa etária pediátrica, os níveis de proteção alcançados com a vacina são de 16 a $40 \%$ após uma única dose, 80 a $95 \%$ depois de duas doses e 98 a $100 \%$, seguindo três doses 6,54 . Nos recém-nascidos prematuros, com menos de $2 \mathrm{~kg}$ de peso, os níveis de anticorpos são mais baixos e as taxas de soroconversão, menores. Estudo recente, realizado por Sadeck \& Ramos ${ }^{56}$, sobre a resposta à vacinação em recém-nascidos prétermo, mostrou uma resposta semelhante àquela dos nascidos a termo. Entretanto, em $25 \%$ daqueles com peso de nascimento igual ou inferior a $1.500 \mathrm{~g}$, a resposta não foi satisfatória. Os autores indicam a necessidade de avaliar a soroproteção após a terceira dose da vacina ou administrar uma dose de reforço aos 12 meses $^{56}$. Entre os adolescentes e os adultos, as taxas de resposta de anticorpos são de 20 a $30 \%$ após uma dose, 75 a $80 \%$ seguindo duas doses e 90 a $95 \%$ depois de três doses ${ }^{6}$. A garantia da eficácia em longo prazo da vacina é devida à resposta anamnéstica anti$\mathrm{HBs}^{4,6,7}$.

Os fatores que diminuem a imunogenicidade da vacina da hepatite $B$, além dos cuidados inadequados com 0 material (cadeia do frio, por exemplo) incluem: idade acima de 40 anos, sexo masculino, tabagismo, obesidade e deficiência imunológica 4,6,7.

Duval et al.57, em estudo prospectivo de 15 anos, compararam a imunogenicidade de duas vacinas pediátricas (Engerix- $B^{\circledR}$ e Recombivax- $B^{\circledR}$ ) e o efeito da dose de reforço dada 5 anos após, em país de baixa endemicidade (Canadá). Observaram que a imunidade persistiu por 5 anos, após a vacinação primária, em $99 \%$ das crianças vacinadas com 8 a 10 anos de idade. Parece haver uma longa proteção (10 a 12 anos) nas crianças de maior risco, vacinadas ao nascer (mães HBsAg positivo e $\mathrm{HBeAg}$ positivo).

Tabela 5 - Preparações e doses recomendadas de vacinas contra hepatite $B$

\begin{tabular}{lcccc}
\hline Vacina & $\begin{array}{c}\text { Idade ou } \\
\text { grupo }\end{array}$ & $\begin{array}{c}\text { Dose } \\
(\boldsymbol{\mu g})\end{array}$ & $\begin{array}{c}\text { Volume } \\
(\mathbf{m L})\end{array}$ & Esquema \\
\hline Engerix-B $^{\circledR} *$ & $0-19$ & 10 & 0,5 & $0,1,6$ \\
Euvax $^{\circledR}+$ & $>20$ & 20 & 1,0 & $0,1,6$ \\
& Diálise & 40 & 2,0 & $0,1,2,6$ \\
Recombivax-HB $^{\circledR} \neq$ & $0-19$ & 5 & 0,5 & $0,1,6$ \\
& $>20$ & 10 & 1,0 & $0,1,6$
\end{tabular}

* GlaxoSmithKline; † Sanofi-Pasteur; ${ }^{\ddagger}$ Merck Sharp and Dohme. 
A duração da imunidade em crianças de baixo risco, nascidas de mães HBsAg negativas, vacinadas logo após o nascimento, ainda está em discussão ${ }^{51}$. Nesses indivíduos, o risco poderia reaparecer durante a adolescência e início da vida sexual.

Petersen et al. 52 estudaram a duração da proteção pósvacina em crianças de alto e baixo risco, com 4 a 13 anos de idade, vacinadas ao nascer. Observaram que, na maioria dos casos, o anti-HBs desaparece em torno dos 5 anos de idade, embora grande parte delas mostrem memória imunológica. Houve falha em $1 / 3$ dessas crianças na resposta anamnéstica após uma dose de reforço. Por outro lado, Gong et al. ${ }^{58}$, na China, avaliaram a proteção da vacina 3 a 12 anos após a vacinação ao nascer, em 2.419 crianças, e concluíram que os efeitos protetores da vacina recombinante foram significativos 12 anos depois. Os autores acreditam não ser necessário reforço da vacina nessas crianças, já que a taxa de positividade do HBsAg não foi ascendente nessa população em anos posteriores ${ }^{58}$.

Zanetti et al.59, na Itália, analisaram a duração da imunidade e a eventual necessidade de dose de reforço em 1.212 crianças e 446 recrutas das forças aéreas, após 10 anos de implementação da vacinação em lactentes e em adolescentes naquele país. Concluíram que uma forte memória imunológica persiste por mais de 10 anos, após um curso primário de imunização, não sendo necessárias doses de reforço da vacina.

Não há dúvida que a imunoprofilaxia é o método de maior custo-benefício para controlar globalmente a infecção pelo vírus $B$ e suas complicações ${ }^{60}$. A imunização passiva, através da imunoglobulina hiperimune (HBIG), provê imunidade temporária, com um custo alto. Por isso, a estratégia mais eficaz tem sido programas de imunização universal para prevenir tanto transmissão perinatal como horizontal da infecção pelo VHB.

As estratégias indicadas para a imunização universal e os grupos de risco são apresentadas a seguir, assim como na Tabela 67,60: crianças de mães HBsAg-positivas; contactantes domiciliares de indivíduos com infecção pelo VHB; trabalhadores com exposição a sangue e/ou hemoderivados; pacientes com transfusões sangüíneas e/ou de hemoderivados freqüentes; freqüentadores de unidades de he- modiálise; apenados em instituições correcionais; homens promíscuos que fazem sexo com homens; pessoas com mais de um parceiro sexual em período de 6 meses; usuários de drogas injetáveis; imunodeprimidos; salvavidas/bombeiros; pessoas originárias de zonas com alta endemicidade; contactantes de imigrantes originários de zonas endêmicas.

Essas estratégias dependem tanto das condições epidemiológicas locais (alta ou baixa prevalência de HBsAg em crianças) quanto dos recursos governamentais para implementação da vacinação. Em áreas onde a prevalência de infecção por VHB é baixa ou os recursos financeiros são limitados, imunização com três doses da vacina, sem triagem pré-natal e sem a administração concomitante de HBIG, é uma estratégia razoável, economiza recursos e tem se mostrado eficaz em países como a Tailândia e em outros países da Ásia60-62.

Nos últimos anos, algumas inovações relacionadas às vacinas vêm sendo avaliadas. Estão sendo testados novas rotas de aplicação, (intranasal, transcutânea, aerossol e oral), novos dispositivos (sistema de microagulhas), finalidade do seu uso (profilático ou terapêutico) e época de administração da vacina ${ }^{63}$. Assim, Patwardahan et al. ${ }^{64}$ testaram, recentemente, a vacinação pré-natal em mães pertencentes a populações de alto risco, e os resultados foram encorajadores quando as mães foram vacinadas na $20^{a}$ e na $24^{a}$ semanas de gestação.

Em estudo recentemente publicado, vários órgãos internacionais, incluindo a OMS e o Centers for Disease Control and Prevention ( $C D C)$, afirmaram que, até o presente momento, não existem dados para concluir que as vacinas, particularmente hepatite $B$, apresentem riscos à saúde, $e$ que não há justificativas para modificar as práticas atuais de imunização ${ }^{65}$. Entretanto, as "cicatrizes" deixadas por relatos irresponsáveis que vinculam efeitos adversos graves às vacinas podem ter um impacto importante na cobertura da imunização.

\section{Vacina HVB em grupos especiais}

É de conhecimento geral que a capacidade imunogênica da vacina é menor em certos grupos de pessoas, entre eles:

Tabela 6 - Estratégias indicadas para imunização universal contra HVB de acordo com os diferentes padrões de endemicidade

\begin{tabular}{lccc}
\hline Prevalência regional & $\begin{array}{c}\text { Screening materno } \\
\text { HbsAg/HBeAg }\end{array}$ & $\begin{array}{c}\text { HBV imunização } \\
\text { HBIG/HBV vacina }\end{array}$ & Custo \\
\hline Alta & Sim/sim & Sim*/sim & Alto \\
Baixa com grupos de alto risco & Sim/não & Sim/sim & Alto \\
Alta & Não/não & Não/sim & Baixo \\
Baixa & Não/não & Não/sim & Baixo
\end{tabular}

* Crianças com mães de alto risco (HBeAg positivo). 
idosos, obesos, alcoolistas, indivíduos submetidos a quimioterapia antiblástica e imunodeprimidos. Para melhorar a resposta à vacina, algumas estratégias podem ser implementadas. Kappor et al.66, por exemplo, usaram o fator estimulante de formação de granulócitos com resultados animadores.

Recém-nascidos prematuros, com menos de $2 \mathrm{~kg}$ ao nascer, devem receber a primeira dose da vacina quando a criança completar 1 mês $^{22}$. A resposta à vacina, nessa circunstância, é comparável à de crianças a termo, independentemente do peso do nascimento e da idade gestacional22.

A imunogenicidade da vacina da hepatite $B$ é baixa em pacientes infectados pelo HIV, mas o esquema ideal de vacinação desses pacientes permanece indefinido. Há limitadas informações sobre a duração da memória imunológica e o momento mais adequado para iniciar a vacinação de pacientes gravemente imunodeprimidos. Sabe-se que a resposta sorológica é diretamente proporcional ao nível de CD4. Estudos conduzidos nas décadas de 1980 e 1990, analisando adultos infectados pelo HIV, demonstraram respostas entre 33 e $56 \%$ a vacinas recombinantes. Adolescentes norte-americanos infectados pelo HIV, vacinados contra hepatite $B$, mostraram uma resposta sorológica diminuída, associada ao aumento de células T (CD8+/ CD38+). Os autores sugerem que a replicação viral continuada e a ativação do sistema imune podem levar à diminuição na capacidade de resposta à vacinação ${ }^{67}$.

Em relação aos hepatopatas crônicos, quando apresentam doença leve ou moderada, os resultados da vacinação são similares aos de adultos saudáveis, atingindo até $100 \%$ de soroconversão em alguns estudos ${ }^{6,17,33}$. Em pacientes com cirrose por álcool, as taxas de soroconversão são baixas, variando de 12 a $75 \%$, e em pacientes em lista de transplante de fígado são ainda mais reduzidas, variando entre 7 e $55 \% 6$.

A vacina contra VHB é recomendada em doses mais altas nos pacientes em hemodiálise. Soroproteção tem sido documentada em $64 \%$ dos pacientes com o esquema normal de vacinação, de três doses, e em $86 \%$ naqueles que recebem uma dose da vacina a mais ${ }^{4,6}$. A resposta depende, basicamente, das doses utilizadas e da fase da doença renal na qual o paciente foi vacinado. Aqueles que apresentam creatinina superior a $4 \mathrm{mg} / \mathrm{dL}$ e estão em diálise respondem de maneira muito inferior àqueles com insuficiência renal mais moderada. Os nefropatas crônicos devem ser vacinados o mais precocemente possível, sendo aconselhável instituir a vacinação antes de submetê-los a diálise. Para esses pacientes, está indicado monitorar os níveis de anticorpos (anti-HBs) anualmente e fornecer uma dose de reforço quando esses níveis estiverem abaixo de $10 \mathrm{mUI} / \mathrm{mL}^{4,6}$.

\section{Impacto da vacinação anti-HVB}

A experiência da imunização contra hepatite $B$ tem sido sempre um sucesso nas campanhas de vacinação universal em diferentes países. É bem conhecido que o êxito não é alcançado quando a estratégia se orienta para vacinar somente grupos de risco. Estima-se que 25 a $30 \%$ das pessoas com hepatite $B$ negue ter qualquer fator de risco para adquirir a infecção e, portanto, não são identificadas como alvos para vacinação. Homens que fazem sexo com homens, usuários de drogas intravenosas e heterossexuais promíscuos são importantes grupos de risco que não costumam ser atingidos nas campanhas vacinais. Nos EUA, somente após a recomendação da vacinação para todos os recém-nascidos em 1991, e da imunização de rotina para adolescentes de 11 e 12 anos, em 1996, é que foi constatada uma redução substancial na incidência da hepatite $B^{6}$.

Uma outra medida fundamental para a redução da incidência das hepatites agudas pelo vírus $B$ foi a recomendação de testar todas as mulheres grávidas para HBsAg e de proteger os neonatos de todas as mães infectadas com vacina e imunoglobulina 4,6,21.

O Programa Nacional de Hepatites, do Ministério da Saúde, está empreendendo uma série de medidas estratégicas para aprofundar o conhecimento relacionado às hepatites no Brasil ${ }^{2}$. Um dos aspectos para os quais está sendo dada relevância especial é a triagem sorológica de HBsAg em gestantes. A importância da hepatite $B$ durante a gestação decorre, principalmente, do alto potencial de transmissão da mãe para o recém-nascido e da tendência de cronificação da hepatite B (70-90\%) quando o contato ocorre em idade precoce.

Um inquérito realizado recentemente para avaliar o conhecimento de ginecologistas e obstetras em relação a diversos aspectos da hepatite $B$ revelou um quadro preocupante ${ }^{68}$. Aproximadamente a metade dos 262 profissionais entrevistados solicitou o marcador (HBsAg) para as mães no primeiro trimestre da gestação, embora se saiba que, para proteger adequadamente o recém-nascido, a triagem deve ser realizada no terceiro trimestre. Por outro lado, a grande maioria dos profissionais (grupo de risco para a hepatite $\mathrm{B}$ ) havia sido vacinada, mas apenas $58 \%$ deles realizaram anti-HBs ${ }^{68}$. O esquema proposto pelo Ministério da Saúde está representado na Figura 2.

O efeito cumulativo, nos EUA, do uso da vacina da hepatite $B$ tem mostrado reduções importantes na incidên-

\section{HBV e triagem gestacional}

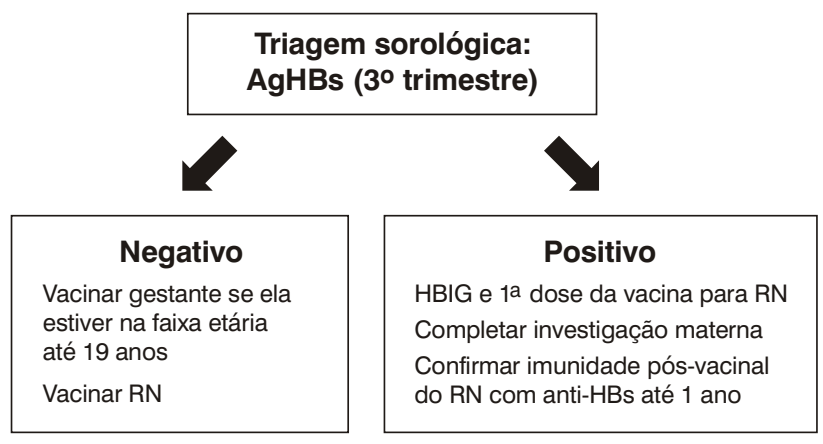

Ministério da Saúde - Brasil.

Figura 2 - Triagem da hepatite B em gestantes 
cia de hepatite aguda B - de 9,2 casos/100.000 em 1981 para 2,8 casos $/ 100.000$ em 2002, sendo que esta última taxa está estável desde 19994,6.

Nos países onde a incidência de hepatite $B$ é baixa, a vacinação universal pode ser considerada uma medida inadequada, do ponto de vista de saúde pública. Nesses casos, a imunização seletiva protegeria a grande maioria dos indivíduos em risco.

Em Formosa, a taxa de mortalidade por hepatite fulminante B em lactentes, entre os anos de 1974 e 1984, antes da era da vacinação universal, era de 5,36/100.000. Essa taxa caiu para 1,71/100.000 entre os anos de 1985 a 1998, após o lançamento do programa de vacinação67. Atualmente, a hepatite fulminante ocorre quase exclusivamente em lactentes infectados de mães portadoras de HBsAg e HBeAg negativo69-72. A vacinação universal contra hepatite $B$, nesses países de alta endemicidade, efetivamente reduziu tanto a transmissão perinatal como a horizontal, diminuindo assim as taxas de hepatite crônica $B^{60,69-71}$. Em Formosa, a incidência de portadores crônicos de HBsAg diminuiu de 10 para $1 \%$ em crianças com menos de 15 anos ${ }^{69}$. Essas diminuições demonstram, claramente, que os programas de vacinação universal são mais eficazes do que a vacinação em grupos de risco.

Atualmente, o desenvolvimento de mutantes, conseqüentes aos programas de vacinação, é a preocupação nesses países de endemicidade alta. A prevalência de mutantes do gene do antígeno de superfície, que pode acarretar falha da vacina, é a preocupação atual, pois aumentou gradualmente 5 a 10 anos após a instituição dos programas $^{60}$. A redução de taxas de infecção pelo HVB, após o início dos programas de vacinação universal, teve um efeito dramático na incidência de carcinoma hepatocelular nas crianças de Formosa. A incidência anual de câncer de fígado diminuiu $75 \%$ - de 0,52/100.000 em crianças nascidas de antes de julho de 1984 para 0,13/100.000 naquelas nascidas após $1984^{72}$. Se essas taxas seguirem declinando assim, uma substancial diminuição de carcinoma hepatocelular também deverá ocorrer em adultos em um futuro próximo.

Por último, é importante lembrar que, em nosso país, a hepatite pelo vírus delta ainda é de alta prevalência em algumas áreas da Amazônia Oriental. Por se tratar de vírus defectivo, a distribuição da infecção superpõe-se à da hepatite B. Ao proteger os indivíduos contra o VHB, estamos também impedindo o espectro de doenças graves determinadas pela associação dos vírus $B$ e delta, tanto de superinfecção como de co-infecção.

\section{Considerações finais}

Infecções causadas por hepatites virais A e B geram problemas enormes relacionados à morbimortalidade, além de custos não só com as doenças, mas também determinam aumento no número de transplantes de fígado. Ao invés de continuarmos pensando nas hepatites $A$ e $B$ como doenças comuns que acometem nossos pacientes, talvez devêssemos encará-las, agora, como infecções que podem ser erradicadas do nosso país. A vacina da HVB já está disponível para todas as crianças quando nascem, para os adolescentes até 19 anos e para todos os pacientes de risco na rede pública. A vacina da HVA, ainda não disponível na rede pública, deve ser indicada para todos os pacientes que têm condições financeiras de recebê-la, pois essas pessoas formam o maior grupo suscetível e, portanto, de maior risco. Lembrando que, para grupos de risco, essa vacina está à disposição, na rede pública, nos CRIE do Brasil.

Os profissionais de saúde e a comunidade leiga, sobretudo os pais e a mídia, devem ser continuadamente informados em relação aos benefícios e uso adequado das vacinas disponíveis no nosso país. As ações empreendidas pela equipe do Instituto Evandro Chagas, na Amazônia, desde 1953, em relação às hepatites virais, devem servir de inspiração a todos nós, pois, apesar do muito que foi feito, é necessário refletir sobre as palavras judiciosas de Bensabath e Soares ${ }^{73}$ : "a tarefa não está concluída".

\section{Conflitos de interesse}

Cristina Targa Ferreira declara que participou de estudos sobre vacinas dos Laboratórios Glaxo e Merck e atualmente participa de um estudo sobre hepatite $C$ (tratamento com IFN peguilado e ribavirina), do Laboratório Roche. Cristina Targa Ferreira e Themis Reverbel da Silveira declaram já ter participado, como pesquisadoras, de estudos dos Laboratórios Roche e GlaxoSmithKline sobre hepatites virais.

\section{Referências}

1. Schiff ER. Introduction. Am J Med. 2005;118 Suppl 10A:1S-6S.

2. Brasil, Secretaria de Políticas de Saúde. Hepatites virais - o Brasil está atento. Normas e manuais técnicos. Brasília: Ministério da Saúde; 2003.

3. Expanded programme on immunization. Global advisory group - Part 1. Wkly Epidemiol Rec. 1992;67:11-5.

4. Center for Disease Control and Prevention (CDC). Hepatitis surveillance report no. 59. Atlanta: US Department of Health and Human Services, Centers for Disease Control and Prevention; 2004. http://www.cdc.gov/ncidod/diseases/hepatitis/resource/ . Acesso: 08/02/2006.

5. Clemens SAC, da Fonseca JC, Azevedo T, Cavalcanti A, Silveira $T R$, Castilho $M C$, et al. Soroprevalência para hepatite $A$ e hepatite B em 4 centros no Brasil. Rev Soc Bras Med Trop. 2000;33:1-10.

6. Davis JP. Experience with hepatitis A and B vaccines. Am J Med. 2005:118 Suppl 10A:7S-15S.

7. Poland GA. Evaluating existing recommendations for hepatitis $A$ and B vaccination. Am J Med. 2005;118 Suppl 10A:16S-20S.

8. Provost PJ, Hilleman MR. Propagation of human hepatitis A virus in cell culture in vitro. Proc Soc Exp Biol Med. 1979;160:213-21.

9. Andre FE. Approaches to a vaccine against hepatitis A: development and manufacture of an inactivated vaccine. J Infect Dis. 1995;171 Suppl 1:S33-9.

10. Andre FE, D'Hondt E, Delem A, Safary A. Clinical assessment of the safety and efficacy of an inactivated hepatitis $A$ vaccine: rationale and summary of findings. Vaccine. 1992;10 Suppl $1: \mathrm{S} 160-8$.

11. Innis $B L$, Snitbhan $R$, Kunasol $P$, Laorakpongse $T$, Poopatanakool $W$, Kozik $C A$, et al. Protection against hepatitis $A$ by an inactivated vaccine. JAMA. 1994;271:1328-34.

12. Werzberger A, Mensch B, Kuter B, Brown L, Lewis J, Sitrin R, et al. A controlled trial of a formalin-inactivated hepatitis $A$ vaccine in healthy children. N Engl J Med. 1992;327:453-7. 
13. Ginsberg GM, Slater PE, Shouval D. Cost-benefit analysis of a nationwide infant immunization programme against hepatitis $A$ in an area of intermediate endemicity. J Hepatol. 2001;34:92-9.

14. Van Damme P, Thoelen S, Cramm M, De Groote K, Safary A, Meheus $A$. Inactivated hepatitis $A$ vaccine: reactogenicity, immunogenicity, and long-term antibody persistence. J Med Virol. 1994;44:446-51.

15. Fujiyama S, Odoh K, Kuramoto I, Mizuno K, Tsurusaki R, Sato T. Current seroepidemiological status of hepatitis $A$ with comparison of antibodies titers after infection and vaccination. J Hepatol. 1994;21:641-5.

16. Clemens R, Safary A, Hepburn A, Roche C, Stanbury WJ, Andre FE. Clinical experience with an inactivated hepatitis A vaccine. J Infect Dis. 1995;171 Suppl 1:S44-9.

17. Keeffe EB, Iwarson S, McMahon BJ, Lindsay KL, Koff RS, Manns $M$, et al. Safety and immunogenicity of hepatitis $A$ vaccine in patients with chronic liver disease. Hepatology. 1998;27:881-6.

18. Van Herck K, Beutels $P$, Van Damme $P$, Beutels $M$, Van den Dries $J$, Briantais $P$, et al. Mathematical models for assessment of long-term persistence of antibodies after vaccination with two inactivated hepatitis A vaccine. J Med Virol. 2000;60:1-7.

19. Cederna JB, Klinzman D, Stapleton JT. Hepatitis A virus-specific humoral and cellular immune responses following immunization with a formalin-inactivated hepatitis $A$ vaccine. Vaccine. $1999 ; 18: 892-8$.

20. Wiedermann G, Kundi M, Ambrosch F, Safary A, D'Hont E, Delem A. Inactivated hepatitis $A$ vaccine: long-term antibody persistence. Vaccine. 1997;15:612-5.

21. Prevention of hepatitis $A$ through active and passive immunization: recommendations of the Advisory Committee on Immunization Practices (ACIP). MMWR Recomm Rep. 1999;48(RR-12):1-37.

22. Mast EE, Margolis HS, Fiore AE, Brinck EW, Goldstein W, Wang $\mathrm{SA}$, et al. A comprehensive immunization strategy eliminate transmission of hepatitis B virus infection in the United States. Recommendations of the Advisory Committee on Immunization Practices (ACIP) - Part 1: Immunization of infants, children, and adolescents. MMWR Recomm Rep. 2005;54:1-31.

23. Hess G, Clemens R, Bienzle U, Schonfeld C, Schunk B, Bock HL. Immunogenicity and safety of an inactivated hepatitis $A$ vaccine in anti-HIV positive and negative homosexual men. J Med Virol. 1995;46:40-2.

24. Gouvea AF, De Moraes-Pinto MI, Ono E, Dinnelli MI, Machado DM, Weckx LY, et al. Immunogenicity and tolerability of hepatitis A vaccine in HIV-infected children. Clin Infect Dis. 2005;41:544-8.

25. Gouvea AF, De Moraes-Pinto MI, Machado DM, Carmo FB, Beltrao SC, Cunegundes KS, et al. Prevalência de anticorpos contra o vírus da hepatite $A$ em crianças e adolescentes expostos e/ou infectados pelo HIV. J Pediatr (Rio J). 2005;81: 205-8.

26. Santagostino E, Gringeri A, Rocino A, Zanetti A, de Biasi R, Mannucci PM. Patterns of immunogenicity of an inactivated hepatitis A vaccine in anti-HIV positive and negative hemophilia patients. Thromb Haemost. 1994;72:508-10.

27. Kemper CA, Haubrich R, Frank I, Dubin G, Buscarino C, McCutchan $J A$, et al. Safety and immunogenicity of hepatitis $A$ in human immunodeficiency virus-infected patients: a double blind randomized, placebo-controlled trial. J Infect Dis. 2003; 187:1327-31.

28. Arslan M, Wiesner RH, Poterucha JJ, Zein NN. Safety and efficacy of hepatitis $A$ vaccination in liver transplantation recipients. Transplantation. 2001;72:272-6.

29. Canero-Velasco MC, Mutti JE, Nucifora S, Alvarez K, Antonuccio MC, Sosa A, et al. Safety and immunogenicity after hepatitis A vaccination in pediatric chronic renal disease patients. J Pediatr Gastroenterol Nutr. 2000;31(Suppl 2):S54.

30. Stark K, Gunther M, Neuhaus R, Reinke P, Schroder K, Linnig S, et al. Immunogenicity and safety of hepatitis $A$ vaccine in liver and renal transplant recipients. J Infect Dis. 1999;180:2014-7.

31. Bell BP. Hepatitis A and hepatitis $B$ vaccination of patients with chronic liver disease. Acta Gastroenterol Belg. 2000;63:359-63.

32. Schwarz KB, Balistreri W. Viral hepatitis. In: American Liver Foundation. Pediatric Liver Research Agenda 2000: a blueprint for the future research goals and strategies to treat prevent and conquer childhood liver diseases. New York: American Liver Foundation; 2000. p. 56-63.

33. Keeffe EB. Acute hepatitis A and B in patients with chronic liver disease: prevention through vaccination. Am J Med 2005:118 Suppl 10A:21S-27S
34. Arguedas MR, Johnson A, Eloubeidi MA, Fallon MB. Immunogenicity of hepatitis $A$ vaccination in decompensated cirrhotic patients. Hepatology. 2001;34:28-31.

35. Ferreira CT, Leite JC, Taniguchi A, Vieira SM, Pereira-Lima J, Silveira TR. Immunogenicity and safety of hepatitis A vaccine in Down syndrome children. J Pediatr Gastroenterol Nutr. 2004;39:337-40.

36. Ferreira CT, da Silveira TR, Vieira SM, Taniguchi AN, PereiraLima J. Immunogenicity and safety of hepatitis $A$ vaccine in children with chronic liver disease. J Pediatr Gastroenterol Nutr. 2003;37:258-61.

37. Ferreira CT, Taniguchi AN, Vieira SM, Pereira-Lima J, da Silveira TR. Prevalência do anticorpo da hepatite A em hepatopatia crônica. J Pediatr (Rio J). 2002;78:503-8.

38. Nebbia G, Giacchino R, Soncini R, Ramaccioni V, Timitilli A, Zanetti AR. Hepatitis A vaccination in chronic carriers of hepatitis B virus. J Pediatr. 1999;134:784-5.

39. McMahon BJ, Beller M, Williams J, Schloss M, Tanttila H, Bulkow L. A program to control an outbreak of hepatitis $A$ in Alaska by using an inactivated hepatitis A vaccine. Arch Pediatr Adolesc Med. 1996;150:733-9.

40. Zamir C, Rishpon S, Zamir D, Leventhal A, Rimon N, Ben-Porath E. Control of a community-wide outbreak of hepatitis $A$ by mass vaccination with inactivated hepatitis A vaccine. Eur J Clin Microbiol Infect Dis. 2001;20:185-7.

41. Tapia-Conyer R, Santos JI, Cavalcanti AM, Urdaneta E, Rivera L, Manterola A, et al. Hepatitis A in Latin America: a changing epidemiologic pattern. Am J Trop Med Hyg. 1999;61:825-9.

42. Acharya SK, Batra Y, Bhatkal B, Ojha B, Kaur K, Hazari S, et al. Seroepidemiology of hepatitis $A$ virus infection among school children in Delhi and north Indian patients with chronic liver disease: implications for HAV vaccination. J Gastroent Hepatol. 2003; 18:822-7.

43. Ferreira CT, Silva GL, Barros FC, Pereira-Lima J. Soroepidemiologia da hepatite $A$ em dois grupos populacionais economicamente distintos de Porto Alegre. GED. 1996;15:85-90.

44. Gomes MAC. Hepatite A: prevalência e fatores de risco na população escolar de São Luís, MA [dissertação]. São Luís: Universidade Federal do Maranhão; 2004.

45. Das A. An economic analysis of different strategies of immunization against hepatitis $A$ virus in developed countries. Hepatology. 1999;29:548-52.

46. Rosenthal P. Cost-effectiveness of hepatitis $A$ vaccination in children, adolescents and adults. Hepatology. 2003;37:44-51.

47. Jacobs RJ, Greenberg DP, Koff RS, Saab S, Meyerhoff AS. Regional variation in the cost effectiveness of childhood hepatitis A immunization. Pediatr Infect Dis J. 2003;22:904-14.

48. Dagan $R$, Leventhal A, Anis $E$, Slater $P$, Ashur $Y$, Shouval D. Incidence of hepatitis $A$ in Israel followings $B$ universal immunization of toddlers. JAMA. 2005;294:202-10.

49. Hanna JN, Hills SL, Humphreys JL. Impact of hepatitis A vaccination of indigenous children on notifications of hepatitis $A$ in north Queensland. Med J Aust. 2004;181:482-5.

50. Bell BP. Hepatitis A vaccine. Pediatr Infet Dis J. 2000;19:1187-8.

51. Ciocca M, Porta G, Silveira TR, Moreira-Silva S. Hepatitis A as an etiological agent of acute liver failure in Latin America. Pediatr Infect Dis J. In press.

52. Petersen KM, Bulkow LR, McMahon BJ, Zanis C, Getty M, Peters $H$, et al. Duration of hepatitis B immunity in low risk children receiving hepatitis $B$ vaccinations from birth. Pediatr Infect Dis J. $2004 ; 23: 650-5$

53. Lok AS. The maze of treatments for hepatitis B. N Engl J Med. 2005;352:2743-6.

54. Shete PB, Daum RS. Real versus theoretical: assessing the risks and benefits of postponing the hepatitis $B$ vaccine birth dose. Pediatrics. 2002;109:701-3.

55. Center for Disease Control and Prevention. Hepatitis B. In: Atkinson W, Hamborsky J, Wolfe C, editors. Epidemiology and prevention of vaccine-preventable diseases. 8th ed. Washington: Public Health Foundation; 2004. p. 191-212.

56. Sadeck LSR, Ramos JLA. Resposta imune à vacinação contra hepatite $B$ em recém-nascidos pré-termo, iniciada no primeiro dia de vida. J Pediatr (Rio J). 2004;80:113-8.

57. Duval B, Gilca V, Boulianne N, De Wals P, Masse R, Trudeau G, et al. Comparative long term immunogenicity of two recombinant hepatitis $B$ vaccines and the effect of a booster dose given after 5 years in a low endemicity country. Pediatr Infec Dis J. 2005; $24: 213-8$. 
58. Gong $X H$, Wang $F Z$, Li $H$, Liu $L R$, Li $Y H$, Wang $B L$, et al. Observation on effects of hepatitis $B$ vaccine immunization for 12 years in children in Beijing. Zhonghua Yu Fang Yi Xue Za Zhi. 2005;39:265-8.

59. Zanetti AR, Mariano A, Romano L, D'Amelio R, Chironna M, Coppola RC, et al. Long-term immunogenicity of hepatitis $B$ vaccination and policy for booster: an Italian multicentre study. Lancet. 2005;366:1379-84.

60. Chang $\mathrm{MH}$. New insights in hepatitis $B$ virus infection. Int Semin Pediatr Gastroenterol Nutr. 2004;12:9-14.

61. Poovorawan Y, Sanpavat S, Pongpunglert W, Chumdermpadetsuk S, Sentrakul P, Vandepapeliere P, et al. Long-term efficacy of hepatitis $B$ vaccine in infants born to hepatitis $B$ e antigenpositive mothers. Pediatr Infect Dis J. 1992;11:816-21.

62. Poovorawan $Y$, Theamboonlers A, Vimolket T, Sinlaparatsamee S, Chaiear K, Siraprapasiri T, et al. Impact of hepatitis B immunisation as part of Thailand's EPI. Vaccine. 2000;19:943-9.

63. Mancini-Bourgine M, Fontaine $H$, Scott-Algara D, Pol S, Brechot $C$, Michel ML. Induction or expansion of $\mathrm{T}$-cell responses by $\mathrm{a}$ hepatitis B DNA vaccine administered to chronic HBV carriers. Hepatology. 2004;40:874-82.

64. Patwardhan $A$, Jamjute $P$, Peter. Foetal immunoprophylaxis with hepatitis $B$ vaccine could be a cost-effective approach towards eradication in the high-risk population: PH2-02. J Pediatr Gastroenterol Nutr. 2005;40:678.

65. François $G$, Duclos $P$, Margolis $H$, Lavanchy $D$, Siegrist $C A$, Meheus $A$, et al. Vaccine safety controversies and the future of vaccination programs. Pediatr Infect Dis J. 2005;24:953-61.

66. Kapoor D, Aggarwal SR, Singh NP, Thakur V, Sarin SK. Granulocyte-macrophage colony-stimulating factor enhances the efficacy of hepatitis B virus vaccine in previously unvaccinated haemodialysis patients. J Viral Hepat. 1999;6:405-9.

67. Wilson CM, Ellenberg JH, Sawyer MK, Belzer M, Crowley-Nowick PA, Puga A, et al. Serologic response to hepatitis B vaccine in $\mathrm{HIV}$ infected and high risk HIV uninfected adolescents in REACH cohort. Reaching for excellence in adolescent care and health. J Adolesc Health. 2001;29(3 Suppl):123-9.
68. Silveira TR, Cunha J, Krebs LS, Ramalho L. Avaliação do grau de conhecimento e de proteção de ginecologistas e obstetras do Rio Grande do Sul em relação à hepatite B. Rev AMRIGS. 2003;47:193-201.

69. Chen HL, Chang CJ, Kong MS, Huang FC, Lee HC, Lin CC, et al. Fulminant hepatic failure in children in endemic areas of hepatitis $B$ virus infection: 15 years after universal hepatitis $B$ vaccination. Hepatology. 2004;39:58-63.

70. Boxall EH, Sira J, Standish RA, Davies P, Sleight E, Dhillon AP, et al. Natural history of hepatitis $B$ in perinatally infected carriers. Arch Dis Child Fetal Neonatal Ed. 2004;89:F456-60.

71. Ni YH, Chang MH, Huang LM, Chen HL, Hsu HY, Chiu TY, et al. Hepatitis $B$ virus infection in children and adolescents in a hyperendemic area: 15 years after mass hepatitis B vaccination. Ann Intern Med. 2001;135:796-800.

72. Chang MH, Chen CJ, Lai MS, Hsu HM, Wu TC, Kong MS, et al. Universal hepatitis $B$ vaccination in Taiwan and the incidence of hepatocellular carcinoma in children. $N$ Engl J Med. 1997;336:1855-9.

73. Bensabath G, Soares MCP. A evolução do conhecimento sobre as hepatites virais na região amazônica: da epidemiologia e etiologia à prevenção. Rev Soc Bras Med Trop. 2004;37(Supl II): 14-36

Correspondência:

Cristina Targa Ferreira

Pedro Weingartner, 105/202

CEP 90430-140 - Porto Alegre, RS

Tel.: (51) 3331.3295

Fax: (51) 3388.4369

E-mail: cristinatarga@terra.com.br 